\title{
Cerebral Microbleeds: A Call for Standardized Advanced Neuroimaging
}

$l^{\mathrm{n}}$ $n$ recent years, cerebral microbleeds have received considerable attention, in part due to the widespread availability of advanced neuroimaging methods for their detection. ${ }^{1,2}$ The prevalence of microbleeds has been well-studied in various diseases and in healthy controls. Their significance, however, is still poorly understood. In stroke and transient ischemic attack, for instance, the key question is whether microbleeds are a marker of increased risk of bleeding due to anticoagulants. Clinical opinion leaders have recently advocated for worldwide collaboration in microbleed research $^{1,2}$ and have recognized the need for standardized imagingacquisition sequences.

Here, we would like to further argue for the use of advanced imaging methods. In a recent meta-analysis, we have shown that the prevalence of microbleeds is influenced by the imaging technique and field strength. ${ }^{3}$ In Alzheimer disease, for example, the prevalence of microbleeds is twice as high with SWI than with conventional gradient-echo MR imaging. Multisite trials and international consortia are going to face a trade-off between standardization and the use of advanced imaging techniques. The maximization of the number of participating sites needs to be weighed against the ability to acquire and analyze SWI data. However, SWI is increasingly adopted as a new standard in many imaging sites. Furthermore, it may be useful to store the unprocessed images of the SWI scans so that they can be processed into quantitative susceptibility maps. ${ }^{4}$ Most important, future advanced processing does not preclude the conventional evaluation of SWI.

Finally, we support the suggestion that microbleeds be recorded electronically with standardized tools. Ideally, the output of such tools should be logfiles that contain microbleed location and certain attributes, as well as images that record the location and size of the microbleeds. These images and logfiles can be easily analyzed across numerous sites; this feature may result in further insight, such as microbleed location in various disease subtypes. Moreover, a low-cost by-product of this approach will be valuable data about the change in the number and size of microbleeds with time. If these imaging data are shared in conjunction with clinical, genetic, or biochemical data, their analysis will provide much

http://dx.doi.org/10.3174/ajnr.A5323 more insight than meta-analyses of aggregate data in the published literature.

In summary, members of the international consortia on microbleeds who will agree on which imaging techniques to use and how to best analyze and share the data may want to consider more advanced data-acquisition techniques and the high likelihood of future progress in quantitative image analysis. The relatively simple gradient-echo imaging can be used on all scanners, but it precludes further quantitative analysis of magnetic susceptibility, with a high false-negative rate. SWI detects much smaller microbleeds than gradient-echo imaging and has the advantage of allowing advanced quantitative analyses to be performed in the future without compromising standardization in the present.

\section{ACKNOWLEDGMENTS}

Alexander Rauscher is funded by Canada Research Chairs. Amir A. Sepehry was funded by the Alzheimer Society of Canada/Canadian Consortium on Neurodegeneration in Aging (ASC/ CCNA). G.-Y. Robin Hsiung is funded by the Ralph Fisher and Alzheimer Society of BC professorship in Alzheimer Disease Research.

Disclosures: G.-Y. Robin Hsiung —UNRELATED: Research grants from Brain Canada, and Canadian Institutes of Health Research; support for travel to the DIAN study and ADNI study investigators meeting; and other funds from Eli Lilly, Biogen, and AstraZeneca for conduction of clinical trials as a site investigator*. *Money paid to the institution.

\section{REFERENCES}

1. Charidimou A, Soo Y, Heo JH, et al; META-MICROBLEEDS Consortium. A call for researchers to join the META-MICROBLEEDS Consortium. Lancet Neurol 2016;15:900 CrossRef Medline

2. Microbleeds International Collaborative Network. Worldwide collaboration in the Microbleeds International Collaborative Network. Lancet Neurol 2016;15:1113-14 CrossRef Medline

3. Sepehry AA, Lang D, Hsiung GY, et al. Prevalence of brain microbleeds in Alzheimer disease: a systematic review and meta-analysis on the influence of neuroimaging techniques. AJNR Am J Neuroradiol 2016;37:215-22 CrossRef Medline

4. Schweser F, Deistung A, Lehr BW, et al. Quantitative imaging of intrinsic magnetic tissue properties using MRI signal phase: an ap- 
proach to in vivo brain iron metabolism? Neuroimage 2011;54:2789807 CrossRef Medline

(D) Rauscher Department of Paediatrics, Division of Neurology D G.-Y.R. Hsiung (1)A.A. Sepehry Department of Medicine, Division of Neurology University of British Columbia Vancouver, British Columbia, Canada 